\title{
What's New in Mother-Infant Vitamin D Deficiency: A 21st Century Perspective
}

\author{
Adekunle Dawodu \\ Global Health Center, Cincinnati Children's Hospital Medical Center, Cincinnati, Ohio, USA
}

\section{Introduction}

Vitamin D is a prohormone and humans depend mostly on its endogenous production to meet the body's requirement. It is usually regarded as essential for calcium and phosphorus homeostasis and bone mineralization. There is increasing interest in vitamin $\mathrm{D}$ because it is now recognized that it has several other physiological functions and its deficiency has been associated with multiple adverse health conditions including autoimmune diseases, certain common cancers, infections and cardiovascular diseases. This perspective provides a brief description of the current knowledge about vitamin D metabolism and functions, addresses various health issues associated with vitamin D status especially in women and children, and discusses new strategies for prevention of vitamin D deficiency in mothers and their infants.

\section{Vitamin D Metabolism and Functions}

Vitamin $\mathrm{D}_{3}$ is formed from epidermal 7-dehydrocholesterol after skin exposure to ultraviolet $\mathrm{B}$ radiation (UVB, wavelength $290-315 \mathrm{~nm}$ ). The amount of UVB available for vitamin $\mathrm{D}_{3}$ synthesis by the skin is dependent on latitude, season, degree of skin pigmentation, duration of skin exposure to sunlight and the use of skin protectors including sunscreens [1]. In comparison to sunlight, diet provides on average less than $10 \%$ of the body's vitamin D requirement. Vitamin D either synthesized in the skin or orally ingested is metabolized in the liver to 25 -hydroxyvitamin $\mathrm{D}$ [25(OH)D], which is used to determine the body's vitamin D status. The $25(\mathrm{OH}) \mathrm{D}$ undergoes transformation in the kidney to form the active metabolite $1,25(\mathrm{OH})_{2} \mathrm{D}$, the hormonal form that regulates calcium and phosphorus homeostasis and bone mineralization [1]. It is now recognized that many other tissues and cells in the body, including the brain, colon, prostate, breast and macrophages, have the potential to locally express the vitamin $\mathrm{D}$ receptor and produce $1,25(\mathrm{OH})_{2} \mathrm{D}$, a potent immunomodulator with the potential to regulate cell growth and differentiation, rennin synthesis and innate immune function [2].

\section{Vitamin D Deficiency and Possible Health Hazards}

Based on physiological and other functional parameters, including intestinal calcium absorption, optimal bone mineral density and fracture prevention in the elderly, serum $25(\mathrm{OH}) \mathrm{D}$ levels $<50 \mathrm{nmol} / \mathrm{l}$ are considered to be vitamin $\mathrm{D}$ deficiency, and levels between 50 and 75 $\mathrm{nmol} / \mathrm{l}$ as vitamin D insufficiency [2]. The cutoff value for children is controversial. Based on the available evidence, the American Academy of Pediatrics considers serum $25(\mathrm{OH}) \mathrm{D}$ concentrations $<50 \mathrm{nmol} / \mathrm{l}$ to be insufficient in infants and children [3]. It is estimated that about 1 billion people worldwide may have vitamin D deficiency or

\section{KARGER \\ Fax +4161306 1234 \\ E-Mail karger@karger.ch}

www.karger.com
(C) 2011 S. Karger AG, Basel

1011-7571/12/0211-0002\$38.00/0

Accessible online at:

www.karger.com/mpp
Adekunle Dawodu, MB, BS

Global Health Center, Cincinnati Children's Hospital Medical Center

3333 Burnet Avenue, MLC 2048

Cincinnati, OH 45229-3039 (USA)

Tel. +1 513636 1966, E-Mail adekunle.dawodu@cchmc.org 
insufficiency [2]. The major causes of vitamin D deficiency in adults are sunshine deprivation and inadequate corrective vitamin D intake. Risk factors for childhood vitamin D deficiency include a lack of sunshine exposure and prolonged breastfeeding without vitamin D supplementation. Maternal vitamin D deficiency is an underrecognized risk factor for vitamin D deficiency at birth and rickets in infancy [4].

In adults, vitamin $\mathrm{D}$ deficiency has been linked with disorders including common cancers, autoimmune, infectious and cardiovascular diseases as has been discussed in a recent excellent review [2]. In addition to rickets, recent studies indicate that childhood vitamin $\mathrm{D}$ deficiency or low intake of vitamin $\mathrm{D}$ is associated with increased risk of infections and autoimmune disease. A study from Ethiopia [5] found that the incidence of rickets is 13 -fold higher among children with pneumonia than controls without pneumonia. Another case control study from India [6] showed that severe acute lower respiratory infections are 11-fold more common in infants with serum $25(\mathrm{OH}) \mathrm{D}$ concentrations $<25 \mathrm{nmol} / \mathrm{l}$ than those with values $>25 \mathrm{nmol} / \mathrm{l}$. In a Finnish birth-cohort study [7], adequate vitamin $\mathrm{D}$ intake in the first year of life was associated with $82 \%$ reduction in the risk of type I diabetes [7]. These findings are consistent with the role of vitamin D in regulating innate and autoimmune responses and highlight the need to evaluate whether or not vitamin D supplementation will reduce the burden of disease.

Serum 25(OH)D concentration $<25 \mathrm{nmol} / 1$ has been reported in upwards of $18-84 \%$ of pregnant women [4] and is associated with increased risk of pregnancy complications and adverse health effects in the developing fetus [8]. A recent review of studies from Europe, the United States, parts of Asia, North Africa and the Middle East [4] indicate that there is a high prevalence of vitamin D deficiency in infants at birth. This is expected because of the strong relationship between maternal and cord blood $25(\mathrm{OH}) \mathrm{D}$ levels. These studies raise the concern that many infants are entering the world with a vitamin D deficit that begins in utero because of maternal vitamin $\mathrm{D}$ deficiency. Globally, there appears to be an increasing prevalence of vitamin D deficiency in infancy, especially among unsupplemented exclusively breast-fed infants, which could result in significant health problems [4]. Despite these reports, there is still a debate about the optimal strategy to prevent childhood vitamin D deficiencies in many populations.

\section{Prevention of Mother-Child Vitamin D Deficiency}

The currently recommended preventive strategy is vitamin D supplementation of $400 \mathrm{IU} /$ day for all exclusively breast-fed infants and other infants with inadequate dietary intake [3]. However, the optimal intake for infants in populations at risk of high prevalence of severe vitamin $\mathrm{D}$ deficiency is unknown. This premise needs to be investigated in large randomized controlled trials. In addition, the associations between childhood and maternal vitamin D deficiency would suggest that in populations where sunshine exposure is limited and vitamin $\mathrm{D}$ intake is poor, vitamin D deficiency is best understood as a maternal and child health problem requiring a strategy to ensure maternal vitamin D sufficiency during pregnancy and lactation [4]. Experts suggest that mothers without adequate sun exposure require up to 1,000 IU vitamin D/ day to prevent vitamin $\mathrm{D}$ deficiency [2]. There are ongoing research studies to determine optimal vitamin $\mathrm{D}$ requirements to promote maternal vitamin D sufficiency and whether maternal supplementation alone will provide vitamin D sufficiency for the mother and her breastfed infant [9].

\section{References}

$\checkmark 1$ Holick MF: Vitamin D: importance in the prevention of cancers, type 1 diabetes, heart disease, and osteoporosis. Am J Clin Nutr 2004;79:362-371.

2 Holick MF: Vitamin D deficiency. N Engl J Med 2007;357:266-281.

3 Wagner CL, Greer FR: Prevention of rickets and vitamin $\mathrm{D}$ deficiency in infants, children, and adolescents. Pediatrics 2008;122: 1142-1152.
4 Dawodu A, Wagner CL: Mother-child vitamin D deficiency: an international perspective. Arch Dis Child 2007;92:737-740.

5 Muhe L, Lulseged S, Mason KE, Simoes EA: Case-control study of the role of nutritional rickets in the risk of developing pneumonia in Ethiopian children. Lancet 1997;349: 1801-1804.

-6 Wayse V, Yousafzai A, Mogale K, Filteau S: Association of subclinical vitamin $\mathrm{D}$ deficiency with severe acute lower respiratory infection in Indian children under 5 years. Eur J Clin Nutr 2004;58:563-567.
7 Hypponen E, Laara E, Reunanen A, Jarvelin MR, Virtanen SM: Intake of vitamin D and risk of type 1 diabetes: a birth-cohort study. Lancet 2001;358:1500-1503.

$\checkmark 8$ Mulligan ML, Felton SK, Riek AE, BernalMizrachi C: Implications of vitamin D deficiency in pregnancy and lactation. Am J Obstet Gynecol 2009;202:429.e1-e9.

$\checkmark 9$ Hollis BW: Vitamin D requirement during pregnancy and lactation. J Bone Miner Res 2007;22(suppl 2):V39-V44. 